\title{
Neonatal adaptation of the immune system Per Brandtzaeg
}

Address: Laboratory for Immunohistochemistry and Immunopathology, Department and Institute of Pathology, University of Oslo, Rikshospitalet-Radiumhospitalet Medical Center, Norway from Fourth Dominique Dormont International Conference. Host-Pathogen Interactions in Chronic Infections Paris, France. 13-15 December 2007

Published: 9 April 2008

Retrovirology 2008, 5(Suppl I):L6 doi: 10.1 I86/I742-4690-5-SI-L6

This abstract is available from: http://www.retrovirology.com/content/5/SI/L6

(C) 2008 Brandtzaeg; licensee BioMed Central Ltd.

\section{Introduction}

The vast majority of immunological challenges confronting the body make contact with mucosal surfaces, including infectious agents and foreign proteins. To maintain homeostasis in the extensive and vulnerable mucosae, they are protected by specialized mechanisms of immune protection. Numerous genes are involved in the regulation of both innate (natural) and adaptive (acquired) immunity, with a variety of modifications introduced over million of years.

\section{Two-layered adaptive mucosal immunity}

In the process of evolution, the mucosal immune system has generated two layers of non-inflammatory defence: (i) immune exclusion performed by secretory IgA antibodies to modulate or inhibit surface colonization of microorganisms and dampen penetration of potentially dangerous soluble factors; and (ii) suppressive mechanisms to avoid local and peripheral hypersensitivity to innocuous antigens, particularly food proteins and components of commensal bacteria. When induced via the gut, the latter phenomenon is called 'oral tolerance', which appears be a rather robust adaptive immune function in view of the fact that large amounts of food proteins pass through the gut, while overt and persistent food allergy is not so common.

\section{Neonatal immune adaptation}

The neonatal period is particularly critical, both with regard to infections and priming for allergic disease. This is so because the mucosal barrier function and the immunoregulatory network are poorly developed for a variable period after birth, and successful neonatal immune adaptation to exogenous stimuli is crucial to health. Notably, immunological homeostasis depends on appropriate microbial colonization as well as adequate timing and dose of foreign food proteins when first introduced in the diet. Dendritic cells are decision makers in the immune system when they perform their antigen-presenting function, thus linking innate and adaptive immunity by sensing the exogenous impact (e.g. conserved microbial molecular patterns) on the mucosa. A balanced indigenous microbiota is required to drive the normal development of both mucosa-associated lymphoid tissue, the epithelial barrier with its secretory IgA system, and mucosally induced tolerance mechanisms such as the generation of regulatory T cells. Moreover, the properties of dendritic cells also appear to be influenced by dietary factors including vitamin A. Other dietary factors such as lipids, particularly the polyunsaturated n-3 fatty acids in fish oil, can likewise in several ways have a beneficial effect on the developing immunophenotype of the infant. The same is true for breast milk, which provides both immunoregulatory factors and secretory antibodies reinforcing the infant's mucosal barrier. 